\title{
Generating structured LPV-models with maximized validity region
}

\author{
Simon Hecker * \\ * Faculty of Electrical Engineering and Information Sciences, Munich \\ University of Applied Sciences, Munich, Germany (Tel: \\ +49-89-1265-3420; e-mail: simon.hecker@hm.edu).
}

\begin{abstract}
A general approach for approximating the nonlinear dynamics of an aircraft model by a linear parameter varying (LPV) model of fixed structure (e.g. affine, polynomial, rational,...) is presented, which is a prerequisite for the application of several LPV based analysis and synthesis techniques. Starting from a given trim-point in the flight envelope, the approach tries to maximize the size of the region (flight envelope) around this trim point, for which the resulting LPV model describes the nonlinear model with sufficient accuracy. For this purpose the sensitivities of the model error with respect to an expansion of the flight envelope in the direction of specific parameters are used to get an optimal overall expansion strategy, which then yields a large validity region for the LPV model.
\end{abstract}

\section{INTRODUCTION}

The synthesis of flight control systems is a challenging task in the presence of various uncertainties in the current flight conditions and aircraft parameters. Although the flight dynamic models are in general nonlinear, frequently linear parameter varying (LPV) approximations [Leith and Leithead, 2000] can be used to describe the dynamics in a continuum of equilibrium points. These models can serve as basis for the synthesis of robust controllers [Magni et al., 1997, Gahinet and Apkarian, 1994], residual generators for fault detection and diagnosis (FDD) [Varga et al., 2011, Henry, 2012], which are aimed to be robust against both measurable and unmeasurable uncertainties.

An aircraft model is typically described by nonlinear differential equations

$$
\begin{aligned}
\dot{x} & =f(x, u, p) \\
y & =g(x, u, p)
\end{aligned}
$$

with state vector $x(t)$ confined to some operating region $X \subset \mathbb{R}^{n}$, input vector $u$ and output vector $y$. This system depends on a parameter vector $p$ (including, e.g. mass $m$, longitudinal position of center of gravity $X_{c g}$, altitude $h$ and calibrated airspeed $V_{\text {cas }}$ ) which is either not exactly known (i.e., uncertain) or is time-varying and belongs to an admissible parameter value set $\Pi$, i.e., $p \in \Pi$.

The nonlinear model (1) is usually approximated by an LPV model of the form

$$
\begin{aligned}
& \dot{\bar{x}}=A(\delta) \bar{x}+B(\delta) \bar{u}, \quad \text { with } \quad S(\delta)=\left[\begin{array}{cc}
A(\delta) & B(\delta) \\
C(\delta) & D(\delta)
\end{array}\right] \\
& \bar{y}=C(\delta) \bar{x}+D(\delta) \bar{u},
\end{aligned}
$$

where the matrices $A(\delta), B(\delta), C(\delta), D(\delta)$ depend rationally on $\delta$ and $\bar{x}, \bar{u}, \bar{y}$ denote deviations of $x, u, y$ from certain trim conditions of the aircraft dynamics. The artificial parameter vector $\delta$ may also depend on state variables, which allows to cover state dependent nonlinearities [Leith and Leithead, 2000] in the representation given by (2). Finally, (2) can be transformed into a Linear Fractional Representation (LFR) [Zhou and Doyle, 1998,
Hecker, 2007, Packard, 1994, Dettori and Scherer, 2000], which is a standard representation in robust control.

To generate the required LPV models we employ a general procedure [Pfifer and Hecker, 2011], starting with the generation of a set of linear, time-invariant (LTI) state-space systems obtained by linearization of (1) at certain equilibrium points (trim points). Least-squares multivariable polynomial fitting is used to interpolate the elements of the state-space matrices and to find a single LPV model (2) approximating the whole set of LTI equilibrium models.

There are LPV synthesis techniques that require an LPV model with affine parametric dependence for the aircraft model, where

$$
\begin{aligned}
& A(\delta)=A_{0}+\delta_{1} A_{1}+\ldots+\delta_{N} A_{N}, \\
& B(\delta)=B_{0}+\delta_{1} B_{1}+\ldots+\delta_{N} B_{N}, \\
& C(\delta)=C_{0}+\delta_{1} C_{1}+\ldots+\delta_{N} C_{N}, \\
& D(\delta)=D_{0}+\delta_{1} D_{1}+\ldots+\delta_{N} D_{N}
\end{aligned}
$$

$\delta_{i}, i=1, \ldots, N$ are the elements of the artificial parameter vector $\delta$. Other methods may allow a polynomial or rational dependence of the system matrices. Usually, a simple (e.g. affine) LPV structure does not allow to cover large regions of the flight envelope with sufficient accuracy. Therefore a new approach was developed, which starts from a given trim-point in the flight envelope and generates an LPV model in a way, such that the region around the trim-point, in which the LPV model accurately describes the nonlinear aircraft behavior, is maximized. In several applications, as for example in fault detection, some fault scenarios may only be relevant in dedicated regions of the flight envelope. This approach then allows to use only a single LPV model during the FDD synthesis process. On the other hand the approach allows to reduce the number of LPV models that are required to cover the full flight envelope as well.

For the generation of an LPV model, efficient polynomial fitting methods as described in [Pfifer and Hecker, 2008, 2011] can be employed and for a given set of grid-point 
LTI models a LPV model with fixed structure is obtained very quick. In general, using a set of LTI models as basis for the LPV model generation may not allow to accurately describe all relevant nonlinear effects. However, in industrial projects the partners only have limited access to confidential, analytic model data and one usual way is to generate LTI models for many points in the flight envelope from a highly accurate nonlinear simulation model. This is especially the case when dealing with aeroelastic aircraft models, which are usually only available for a set of mass and center of gravity cases. Nevertheless, successful projects in the area of flight control [Magni et al., 1997], clearance of flight control laws [Varga et al., 2012] and fault detection [Varga et al., 2011] have shown that LPV modeling based on LTI grid point models is a very useful approach.

\section{GENERATION OF THE FIXED STRUCTURE LPV MODEL}

In this section we will present a general process for generating a LPV model, where the region of the flight envelope, in which the model accurately describes the nonlinear aircraft behavior is maximized. As a first step a very accurate but more complex (e.g. not affine) LPV model is generated using existing methods [Pfifer and Hecker, 2011], which serves as a basis for the generation of the simpler (affine, polynomial, rational,...) LPV model. The reason for this 'intermediate' model is that the process for generating the simple model requires the calculation of a huge set of LTI grid point models within the flight envelope. With the original nonlinear aircraft model, generating an LTI grid point model requires to perform trimming and linearization. Using efficient software tools from DLR (German Aerospace Center), these steps still take several seconds computation time for each grid point, which obviously limits the number of grid points that can be calculated in a reasonable time. On contrary, from the 'intermediate' LPV model an LTI grid-point model can be obtained by evaluating (2) at given parameter values, allowing to generate hundreds of LTI models within seconds.

Of course the final validation of the simple LPV model has to be performed with the original nonlinear aircraft model, but a least for the ADDSAFE ${ }^{1}$ model the validation results with the accurate 'intermediate' LPV model almost coincided with the results using the original nonlinear model.

Remark 1. One may criticize that we generate the final (fixed structure) LPV model from another (intermediate) LPV model, which is already an approximation of the original nonlinear model. In fact one may generate additional model errors by doing this, however already the nonlinear model description neglects many real world dynamics and includes a huge amount of simplifications. The advantage in terms of computation time for generating LTI models was more important for us than using the more accurate model and the results have shown that the final model is still sufficiently accurate to be used for FDD synthesis techniques.

\footnotetext{
1 ADDSAFE is the acronym of the European research project Advanced Fault Diagnosis for Safer Flight Guidance and Control - AAT-2008-RTD-233815 (2009-2012).
}

\subsection{Generation of an 'intermediate' accurate LPV model}

The basis for the following steps to generate a fixed structure LPV model, is a quasi-LPV model $S(\delta)$ as in (2), which can for instance be obtained using the methods described in [Pfifer and Hecker, 2011] or via a function substitution technique as introduced by [Tan, 1997]. The resulting quasi-LPV system (2) usually does not depend polynomially on the parameter vector $\delta$, but includes rational or more general nonlinear functions. More precisely, there are $\left\{s_{i}(\delta)\right\}_{1}^{q}$ elements in $S(\delta)$ which need to be approximated by simpler (e.g. affine or polynomial) functions, in order to transform $S(\delta)$ into a fixed structure LPV model $\widetilde{S}(\delta)$. In a typical aerospace application the set $\left\{s_{i}(\delta)\right\}_{1}^{q}$ would contain for example the aerodynamic forces and moments coefficients.

In the following process we will generate a grid of values $s_{i, k}$ for each function $s_{i}$ at a set of pre-specified parameter values. The value $s_{i, k}$ represents the $i^{\text {th }}$ function evaluated at the $k^{\text {th }}$ point and $\delta^{(k)}$ is the corresponding parameter vector. For each index value $k$ an LTI system with transfer function $G_{k}(s):=C\left(\delta^{(k)}\right)\left(s I-A\left(\delta^{(k)}\right)\right)^{-1} B\left(\delta^{(k)}\right)+D\left(\delta^{(k)}\right)$ can be built by evaluating the quasi-LPV model (2) at $\delta=\delta^{(k)}$.

Note, that the 'intermediate' LPV model $S(\delta)$ may also be valid only in some part of the flight envelope. However, given the same model accuracy, due to the simpler structure, the validity region of the fixed structure LPV model will typically be smaller. At least for the ADDSAFE project, the fault cases were usually specified for a certain trim-point within the flight envelope and $S(\delta)$ could always be generated such that a very large region of the flight envelope around this specified trim-point was accurately covered.

For a given aircraft model $\delta$ is the vector of aircraft and flight condition parameters including e.g. the calibrated airspeed $V_{\text {cas }}$, the aircraft altitude $h$, the aircraft mass $m$ and the position of the center of gravity $X_{c g}$ along the $x$-axis. In the following we assume that $S(\delta)$ is valid in a parameter region $\Pi=\left\{\delta: \delta_{i} \in\left[\delta_{i, \min }, \delta_{i, \max }\right], i=\right.$ $1, \ldots, N\}$ and without loss of generality we assume that the parameters are normalized, i.e. $\delta_{i, \min }=-1, \delta_{i, \max }=1$. Furthermore we assume that the nominal value $\delta_{\text {nom }}=$ $\left(\delta_{1, n o m}, \ldots, \delta_{N, n o m}\right)$ defining a specific flight case is centered in the validity region, i.e. $\delta_{i, \text { nom }}=0$.

\subsection{Grid-point based generation of fixed structure LPV models and validity regions}

For the generation of the fixed structure LPV model $\widetilde{S}(\delta)$, the first step is to calculate a set of grid-point models $\left\{S_{k}\right\}_{1}^{m}$ by evaluating $S(\delta)$ at some pre-specified grid-points $\delta^{(k)}, k=1, \ldots, m$. To calculate $\widetilde{S}(\delta)$, again the methods described in [Pfifer and Hecker, 2011] are employed, where one can easily force the structure of the resulting LPV model to be for example affine. Besides the fixed structure LPV model

$$
\widetilde{S}(\delta)=\left[\begin{array}{ll}
\widetilde{A}(\delta) & \widetilde{B}(\delta) \\
\widetilde{C}(\delta) & \widetilde{D}(\delta)
\end{array}\right]
$$


the methods also deliver a measurement of the distance (error) $d$ between the very accurate LPV model $S(\delta)$ and $\widetilde{S}(\delta)$ at the given grid-points $\delta^{(k)}$ with

$$
d=\max _{k} \nu\left(G_{k}, \widetilde{G}_{k}\right), \quad k=1, \ldots, m,
$$

where $\widetilde{G}_{k}(s)=\widetilde{C}\left(\delta^{(k)}\right)\left(s I-\widetilde{A}\left(\delta^{(k)}\right)\right)^{-1} \widetilde{B}\left(\delta^{(k)}\right)+\widetilde{D}\left(\delta^{(k)}\right)$ is the transfer function of $\widetilde{S}(\delta)$ at a given grid point $\delta=\delta^{(k)}$. $\nu$ denotes the $\nu$-gap metric [Vinnicombe, 1993] and is chosen as a measurement for the distance between the two models. The $\nu$-gap metric can take values between zero and one with zero meaning that two plants match exactly and one that they are far apart. The $\nu$-gap metric has a decisive advantage over other system norms, though, as an error measurement for LPV model generation: It is also defined for unstable systems. Since in many practical cases the plant may be at least slightly unstable (e.g., the phugoid mode) in the admissible parameter set, special care has to be taken when choosing other system norms.

For a given set of $m$ grid-point LTI models the calculation of $\widetilde{S}(\delta)$ and the distance $d$ is straightforward and can be done very quickly. However, the main question is how to choose the grid-points or more importantly how to add new grid-points, such that the size of the region $\widetilde{\Pi}=\{\delta$ : $\left.\delta_{i} \in\left[\widetilde{\delta}_{i, \min }, \widetilde{\delta}_{i, \max }\right], i=1, \ldots, q\right\} \subset \Pi$ of the flight envelope where $\widetilde{S}(\delta)$ accurately describes $S(\delta)$ - and therefore also the original nonlinear aircraft model - is maximized.

For each parameter interval $\delta_{i} \in\left[\widetilde{\delta}_{i, \min }, \widetilde{\delta}_{i, \max }\right]$ we have $m_{i}$ grid-points and we assume that the numbers $m_{i}$ are sufficiently large to accurately describe the aircraft model dynamics in $\widetilde{\Pi}$. The overall number of grid-points $m$ is given by the product of the numbers $m_{i}, i=1, \ldots, q$ of grid-points for each parameter, i.e. $m=m_{1} \cdot m_{2} \cdot \ldots \cdot m_{q}$. Of course the choice of $m_{i}$ and also the placement (equidistant or not) of the $m_{i}$ grid-points within a parameter interval highly depends on the nonlinear model and can usually be done only with a very good knowledge of the model. At the beginning we will usually start with $m_{i}=1$ and $\widetilde{\Pi}=\delta_{n o m}=0$, i.e. our initial validity region is given by the nominal flight point.

\subsection{Calculation of sensitivities}

For each parameter $\delta_{i}, i=1, \ldots, q$, we can now define the validity regions $\widetilde{\Pi}_{\epsilon_{i}}$, where for example

$$
\begin{aligned}
\widetilde{\Pi}_{\epsilon_{1}}=\left\{\delta: \delta_{1}\right. & \in\left[\widetilde{\delta}_{1, \min }, \widetilde{\delta}_{1, \max }+\epsilon\right], \\
\delta_{i} & \left.\in\left[\widetilde{\delta}_{i, \min }, \widetilde{\delta}_{i, \max }\right], i=2, \ldots, q\right\},
\end{aligned}
$$

i.e. $\widetilde{\Pi}_{\epsilon_{1}}$ is obtained from $\widetilde{\Pi}$ by increasing the parameter interval for $\delta_{1}$ by a small amount $\epsilon$. In terms of gridpoints the region $\widetilde{\Pi}_{\epsilon_{1}}$ is described by adding the grid-point $\delta_{1}=\widetilde{\delta}_{1, \max }+\epsilon$ to the $m_{1}$ already existing grid-points for $\delta_{1}$ resulting in an overall number of $m_{\epsilon_{1}}=\left(m_{1}+1\right) \cdot \ldots \cdot m_{q}$ grid-points for the description of $\widetilde{\Pi}_{\epsilon_{1}}$.

Fixed structure LPV models $\widetilde{S}_{\epsilon_{i}}, i=1, \ldots, q$ and distances $d_{\epsilon_{i}}$ can then be calculated for the regions $\widetilde{\Pi}_{\epsilon_{i}}$ described by the corresponding $m_{\epsilon_{i}}$ grid-points. Based on this data we can calculate

$$
\Delta_{i}=d_{\epsilon_{i}}-d,
$$

as an approximation of the sensitivity of the model error $d$ with respect to an expansion of the validity interval of a single parameter $\delta_{i}$.

The values $\Delta_{i}$ are a good indicator of the importance and nonlinearity of a parameter in describing the behavior of the nonlinear model and a large value of $\Delta_{i}$ will yield a 'large' loss of model accuracy, when expanding the validity region $\widetilde{\Pi}$ in this direction.

\subsection{Sensitivity-based expansion of validity region}

The goal is now to find a fixed structure LPV model that represents the nonlinear model accurately within a validity region of maximum size. In our case, the size $V$ of the validity region is defined by the volume of the hyperrectangular given as

$$
V:=\prod_{i=1}^{q}\left(\widetilde{\delta}_{i, \max }-\widetilde{\delta}_{i, \min }\right),
$$

and $V$ will be expanded in an optimal way until the distance $d$ reaches a pre-defined maximum value $d_{\max }$, guaranteeing a desired accuracy of the affine model.

Starting from an initial validity region $\widetilde{\Pi}=\delta_{\text {nom }}=0$, with $\widetilde{\delta}_{i, \min }=\widetilde{\delta}_{i, \max }=0, i=1, \ldots, q$ and $m_{i}=1$, a usual way is to equally expand the validity region by a small amount $l$ in all positive and negative parameter directions, which can be described by the following steps:

(1) Add two grid-points (to the already existing $m_{i}$ gridpoints for each parameter) with values $\widetilde{\delta}_{i, \min }-l$ and $\widetilde{\delta}_{i, \max }+l$ for each parameter, such that the new validity region is given by

$$
\widetilde{\Pi}=\left\{\delta: \delta_{i} \in\left[\widetilde{\delta}_{i, \min }-l, \widetilde{\delta}_{i, \max }+l\right], i=1, \ldots, q\right\} .
$$

(2) Calculate $\widetilde{S}(\delta)$ and $d$ for the expanded validity region. (3) If $d \geq d_{\max }$ then stop, else go to step 1.

The resulting LPV model is then valid within a region, where all parameter intervals $\left[\widetilde{\delta}_{i, \min }, \widetilde{\delta}_{i, \max }\right], i=1, \ldots, q$ are of equal size, which usually does not yield the maximum size $V$ for the validity region. To maximize $V$ we propose a sensitivity based expansion of the validity region described by the following steps:

(1) For each parameter $\delta_{i}$ calculate the sensitivities $\Delta_{i}$ as described in section 2.3.

(2) Add two grid-points with values $\widetilde{\delta}_{i, \min }-l / \Delta_{i}$ and $\widetilde{\delta}_{i, \max }+l / \Delta_{i}$ for each parameter, such that the new validity region is given by

$$
\begin{array}{r}
\widetilde{\Pi}=\left\{\delta: \delta_{i} \in\left[\widetilde{\delta}_{i, \min }-\frac{l}{\Delta_{i}}, \widetilde{\delta}_{i, \max }+\frac{l}{\Delta_{i}}\right],\right. \\
i=1, \ldots, q\} .
\end{array}
$$

(3) Calculate $\widetilde{S}(\delta)$ and $d$ for the expanded validity region.

(4) If $d \geq d_{\max }$ then stop, else go to step 1.

Sketch of a proof: For a model with parameters $\delta_{1}, \ldots, \delta_{N}$ it is shown that the sensitivity based expansion yields a maximum size $V$. Starting from the nominal flight point $(\widetilde{\Pi}=0)$ and given the sensitivities $\Delta_{1}, \ldots, \Delta_{N}$, the size $V$ after one expansion step will be $V=2^{N} \cdot l_{1} \cdot \ldots \cdot l_{N}$, 
where $l_{i}, i=1, \ldots, N$ are the expansions for the parameter intervals yielding

$$
\widetilde{\Pi}=\left\{\delta: \delta_{i} \in\left[-l_{i}, l_{i}\right] .\right\}
$$

For small values $l_{i}$ it is assumed that the distance $d$ after one expansion step is given by

$$
d=2 \cdot \sum_{i=1}^{N} \Delta_{i} \cdot l_{i}=2 \cdot d_{f}
$$

where $2 \cdot d_{f}$ is a fixed distance that should be reached after one expansion step. The question is to find optimal values $l_{i}$ such that $V$ is maximized after this expansion step. For this purpose one can solve (7) for $l_{1}$ yielding

$$
l_{1}=\frac{d_{f}-\sum_{i=2}^{N}\left(\Delta_{i} \cdot l_{i}\right)}{\Delta_{1}}
$$

and with

$$
L=\prod_{i=2}^{N} l_{i}
$$

the size $V$ is given as

$$
V=\frac{2^{N}}{\Delta_{1}} \cdot\left(d_{f} \cdot L-\sum_{i=2}^{N}\left(l_{i} \cdot \Delta_{i} \cdot L\right)\right) .
$$

Differentiating (8) with respect to $l_{j}, j=2, \ldots, N$ yields

$$
\frac{\partial V}{\partial l_{j}}=\frac{2^{N}}{\Delta_{1}} \cdot\left(d_{f} \cdot \frac{L}{l_{j}}-2 \cdot L \cdot \Delta_{j}-\sum_{i \neq j, i=2}^{N}\left(\frac{L}{l_{j}} \cdot l_{i} \cdot \Delta_{i}\right)\right),
$$

which is zero for $l_{i}=\frac{l}{\Delta_{i}}, i=2, \ldots, N$ with $l=d_{f} / N$. One can easily show that this is the solution for the global maximum of $V$ and with (7) this also yields $l_{1}=\frac{l}{\Delta_{1}}$, such that all $l_{i}, i=1, \ldots, N$ correspond to the expansion strategy proposed above.

Example 1. Consider the two parametric system with

$$
\begin{aligned}
A(\delta) & =\left[\begin{array}{c|c}
0 & 1 \\
\hline-c / m & -d / m
\end{array}\right], B(\delta)=\left[\frac{0}{1 / m}\right], \\
C & =\left[\begin{array}{ll}
1 & 0
\end{array}\right], D=0,
\end{aligned}
$$

which may represent a spring, mass, damper system with stiffness $c=2+\delta_{1}$, mass $m=2+\delta_{2}$ and damping coefficient $d=1$. This system depends rationally on the parameters $\delta_{1}$ and $\delta_{2}$. Approximating (9) with an affine LPV system with maximum error $d_{\max }=0.14$ yields a validity region

$$
\widetilde{\Pi}=\left\{\delta: \delta_{1} \in[-0.49,0.49], \delta_{2} \in[-0.49,0.49]\right\}
$$

when using the equal expansion and

$$
\widetilde{\Pi}=\left\{\delta: \delta_{1} \in[-0.86,0.86], \delta_{2} \in[-0.35,0.35]\right\}
$$

using the sensitivity based expansion. The sizes of the validity regions are given by $0.98^{2}=0.9604$ and 0.7 . $1.72=1.204$ and the second one is around $25 \%$ larger. For the calculation of the sensitivities $\epsilon=0.1$ was used and during all expansion steps the sensitivities with respect to $\delta_{2}$ were between 2 and 3 times larger than the sensitivities for $\delta_{1}$, thus yielding a larger interval for $\delta_{1}$.

\subsection{Implementation aspects}

An open question when implementing the sensitivity based expansion strategy is the choice of $l$. In case of four parameters $\delta_{i}$ and assuming that $l$ is small, the distance $d$ after one expansion step is approximately given by $d=2\left(\sum_{i=1}^{N} \Delta_{i} \cdot l / \Delta_{i}\right)=2 \cdot N \cdot l$. Given a maximum allowable distance $d_{\max }$ as the final required accuracy of the (affine) fixed structure LPV model, at least for the ADDSAFE model a good choice was $l=d_{\max } /(8 \cdot N)$, i.e. it requires about 4 expansion steps until the maximum allowable distance $d_{\max }$ is reached. The overall number of grid points will then be $8^{N}$ for the generation of the final LPV model. In case of four parameters this will be 4096 grid points, which could still be solved within seconds. However, due to this exponential growth of the number of grid points the method may be restricted to a small number of parameters.

Using the proposed strategy it may happen that the final parameter intervals for parameters with large sensitivities are small, i.e. the fixed structure LPV model only covers a very small region for this parameter in the flight envelope. In order to enforce the expansion in these directions one may easily introduce weighting parameters $w_{i}$ such that the validity region after one expansion step is given by

$$
\begin{array}{r}
\widetilde{\Pi}=\left\{\delta: \delta_{i} \in\left[\widetilde{\delta}_{i, \min }-\frac{l \cdot w_{i}}{\Delta_{i}}, \widetilde{\delta}_{i, \max }+\frac{l \cdot w_{i}}{\Delta_{i}}\right],\right. \\
i=1, \ldots, q\} .
\end{array}
$$

Choosing a value $w_{i}>1$ will then yield a larger validity region for the parameter $\delta_{i}$ for the final fixed structure LPV model. As a consequence the other parameter intervals will then be smaller and it requires some trials to choose the $w_{i}$ in order to form the resulting validity region in a desired way.

\section{RESULTS}

The proposed sensitivity based expansion was applied for various flight cases of the ADDSAFE benchmark model. To show the advantage of the proposed method we generated an affine LPV model for the complete aircraft dynamics with two parameters, the aircraft mass $m$ and the $x$ position of the center of gravity $X_{c g}$. We started with an initial validity region (gray area) as shown on top of Figure 1. Choosing the strategy of equally expanding the validity region with respect to $m$ and $X_{c g}$, we reached a given distance $d_{\max }$ of the affine LPV model for the validity region as shown in the middle part of Figure 1. When choosing the sensitivity-based expansion, the same distance $d_{\max }$ was reached for the validity region as shown in the lower part of Figure 1. Comparing the area of the two regions, the second one is around $50 \%$ larger, i.e. the sensitivity based expansion allows to obtain an affine LPV model that is valid in a larger region of the weight and balance domain of the aircraft. This shows that for this region, the dependence of the nonlinear model on $X_{c g}$ can be better approximated with an affine parameter dependence than the dependence on $m$. This results in a larger sensitivity for $m$ and therefore in a smaller expansion, when using the sensitivity based strategy.

For all the considered flight cases within the ADDSAFE project the sensitivity based expansion strategy always allowed to obtain larger validity regions compared to an equal expansion strategy. In average the validity regions were around $25 \%$ larger. For the full model with four 

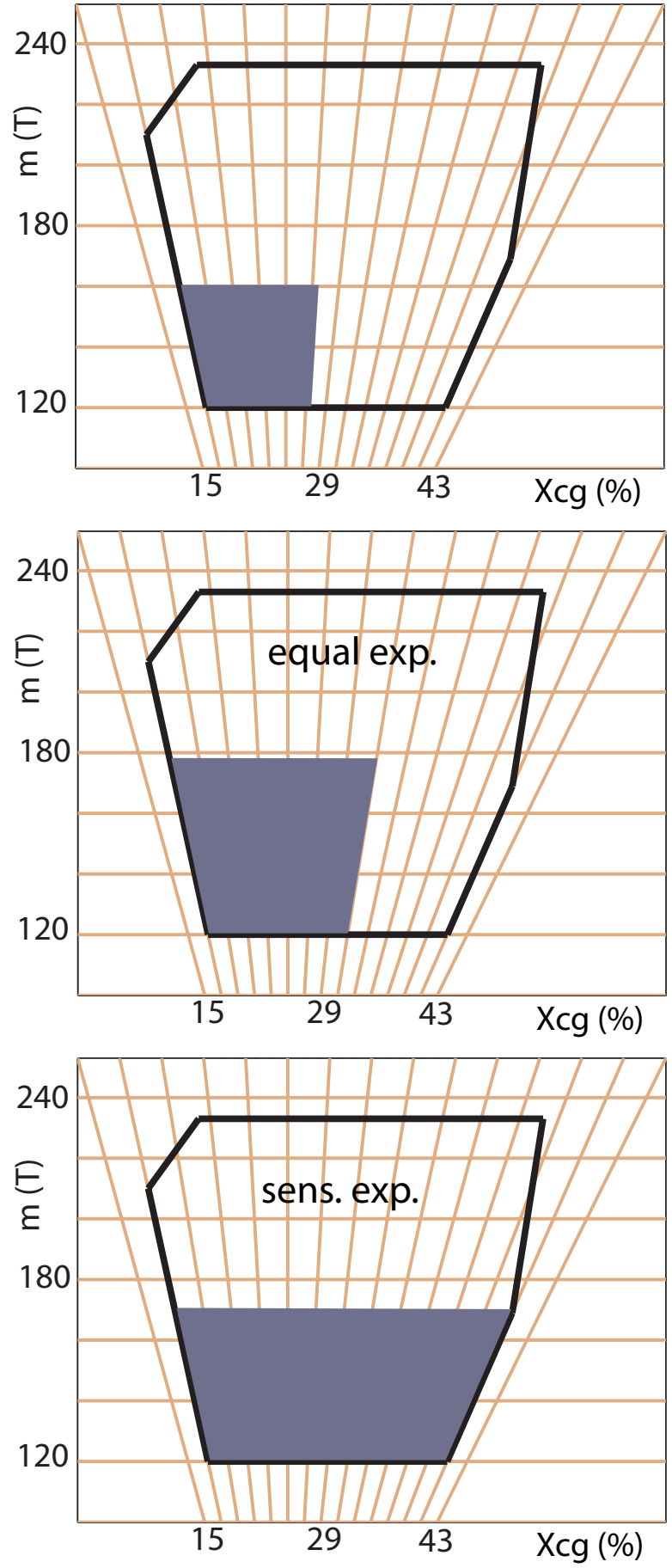

Fig. 1. Achieved validity regions

parameters, the largest sensitivities (and smallest validity intervals) were usually obtained for the calibrated airspeed $V_{\text {cas }}$ and the altitude $h$.

As maximum allowable distance we have usually chosen $d_{\max }=0.2$, and all the resulting affine LPV models were also validated using simulation. Therefore a small (2030) random set of trim-points was chosen for which the nonlinear model was compared to the LPV model using elevator, rudder and aileron step inputs. Figure 2 shows one example of such a simulation, were the green lines correspond to the nonlinear model and the blue lines show
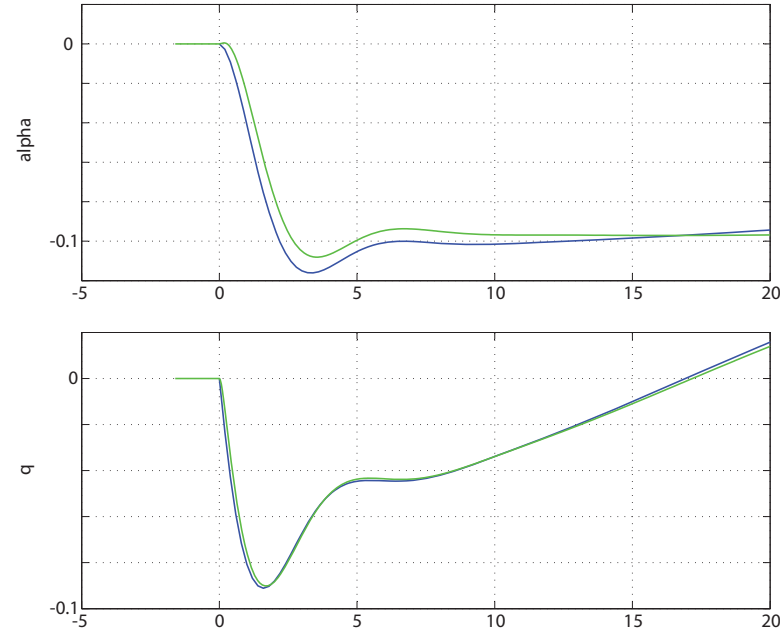

Fig. 2. Simulation: elevator step input

the angle of attack $\alpha$ and the pith rate $q$ for an elevator step input of the affine LPV model.

\section{CONCLUSION}

For the full aircraft dynamics of the ADDSAFE model the requirement was to develop an affine LPV model that is valid for a certain flight case (trim-point) and if possible also in a (large) flight domain around this trim-point. For this purpose we developed a strategy that allows to generate a LPV model of fixed structure, such that the validity region around a certain nominal trim-point in which the LPV model accurately represents the nonlinear aircraft dynamics is maximized. The strategy is based on the sensitivities of the model error with respect to the direction in which the validity region is expanded and allowed to obtain validity regions that were in average around $25 \%$ larger than regions obtained with a standard strategy.

\section{REFERENCES}

M. Dettori and C. Scherer. New robust stability and performance conditions based on parameter dependent multipliers. In Proceedings of IEEE Conference on Decision and Control, 2000.

P. Gahinet and P. Apkarian. A linear matrix inequality approach to $H_{\infty}$ control. Inter. Journ. of Robust \& Nonlinear Control, 1994.

S. Hecker. Generation of low order LFT Representations for Robust Control Applications. VDI Verlag, 2007.

D. Henry. Structured fault detection filters for LPV systems modeled in an LFR manner. International Journal of Adaptive Control and Signal Processing, 26: 190-207, 2012.

D.J. Leith and W.E. Leithead. Survey of gain-scheduling analysis and design. International Journal of Control, 73(11):1001-1025, 2000.

J.F. Magni, S. Bennani, and J. Terlouw, editors. Robust Flight Control: A Design Challenge, Lecture Notes in Control and Information Sciences (224). Springer, 1997.

A. Packard. Gain scheduling via linear fractional transformations. System and Control Letters, 22:79-92, 1994.

H. Pfifer and S. Hecker. Generation of optimal linear parametric models for LFT-based robust stability analysis 
and control design. In Proceedings of IEEE Conference on Decision and Control, 2008.

H. Pfifer and S. Hecker. Generation of optimal linear parametric models for LFT-based robust stability analysis and control design. IEEE Transaction on Control Systems Technology, 19, 2011.

W. Tan. Applications of linear parameter varying control theory. Master's thesis, University of California at Berkeley, 1997.

A. Varga, S. Hecker, and D. Ossmann. Diagnosis of actuator faults using LPV-gain scheduling techniques. In Proceedings of the AIAA Conference on Guidance, Navigation and Control, Portland, USA, 2011.

A. Varga, A. Hansson, and G. Puyou, editors. Optimization Based Clearance of Flight Control Laws. Springer, 2012.

G. Vinnicombe. Frequency domain uncertainty and the graph topology. IEEE Transactions on Automatic Control, 38, 1993.

K. Zhou and J. C. Doyle. Essentials of Robust Control. Prentice Hall, New Jersey, 1998. 